Research Article

\title{
Reliability Analysis for Bypass Seepage Stability of Complex Reinforced Earth-Rockfill Dam with High-Order Practical Stochastic Response Surface Method
}

\author{
Jinwen He $\mathbb{D}^{1,2,3}$ Ping Zhang $\mathbb{D}^{2},{ }^{2}$ and Xiaona $\mathrm{Li} \mathbb{D}^{4}$ \\ ${ }^{1}$ Hubei Key Laboratory of Disaster Prevention and Mitigation, China Three Gorges University, Yichang, Hubei 443002, China \\ ${ }^{2}$ College of Hydraulic \& Environmental Engineering, China Three Gorges University, Yichang, Hubei 443002, China \\ ${ }^{3}$ Research Center on Water Engineering Safety and Disaster Prevention of the Ministry of Water Resources, \\ Yangtze River Scientific Research Institute, Wuhan, Hubei 430010, China \\ ${ }^{4}$ School of Water Resources and Hydroelectric Engineering, Xi'an University of Technology, Xi'an 710048, China \\ Correspondence should be addressed to Jinwen He; hejinwen2004@126.com
}

Received 23 April 2019; Revised 4 August 2019; Accepted 16 September 2019; Published 24 October 2019

Academic Editor: Federica Caselli

Copyright (c) 2019 Jinwen He et al. This is an open access article distributed under the Creative Commons Attribution License, which permits unrestricted use, distribution, and reproduction in any medium, provided the original work is properly cited.

\begin{abstract}
Practical stochastic response surface method (SRSM) using ordinary high-order polynomials with mixed terms to approximate the true limit state function (LSF) is proposed to analyze the reliability of bypass seepage stability of earth-rockfill dam. Firstly, the orders of random variable are determined with a univariate fitting. Secondly, nonessential variables are excluded to identify possible mixed terms. Thirdly, orthogonal table is used to arrange additional samples, and stepwise regression is conducted to achieve a specific high-order response surface polynomial (RSP). Fourthly, Monte Carlo simulation (MCS) is used to calculate the failure probability, and RSP is updated by arranging several additional samplings around the design point. At last, the Bantou complex reinforced earth-rockfill dam was taken as an example. There are 6 random variables, that is, the upper water level and 5 hydraulic conductivities (HCs). The result shows that a third-order RSP can ensure good precision, and the failure probability of bypass seepage stability is $3.680 \times 10^{-5}$ within an acceptable risk range. The $\mathrm{HC}$ of concrete cut-off wall and the $\mathrm{HC}$ of rockfill are unimportant random variables. Maximum failure probability at the bank slope has positive correlation with the HC of curtain and the upper water level, negative correlation with the $\mathrm{HC}$ of alluvial deposits, and less significance with the $\mathrm{HC}$ of filled soil. With the increase of coefficient of variation (Cov) of the HC of curtain, the bypass seepage failure probability increases dramatically. Practical SRSM adopts a nonintrusive form. The reliability analysis and the bypass seepage analysis were conducted separately; therefore, it has a high computational efficiency. Compared with the existing SRSM, the RSP of practical SRSM is simpler and the procedure of the reliability analysis is easier. This paper provides a further evidence for readily application of the high-order practical SRSM to engineering.
\end{abstract}

\section{Introduction}

With a long-term operation of earth-rockfill dams, structural failure caused by seepage may make a dam destroy [1]. About 25\% of the damage of earth-rockfill dams is caused by seepage failure, worldwide [2], while with a rate of $30 \%$ in China [3]. In recent years, a large number of earth-rockfill dams have been reinforced in China by adding a new concrete cut-off wall or an impervious curtain [4]. Although the waterhead in the downstream is dropped, the bypass seepage gradient still plays a controlling role $[5,6]$, so that the bypass seepage analysis should be considered as a $3 \mathrm{D}$ problem. At present, a seepage gradient less than an allowable value is adopted to represent the instability criteria of seepage failure, but the deterministic analysis cannot reflect the parameter uncertainty of bypass seepage effectively.

Many investigators have contributed to the reliability analysis of seepage stability. Wei and Shen [7] combined the hydraulic method and Rackwitz-Fiessler method (R-F or JC 
method) to analyze the seepage stability of a homogeneous earth dam. Li et al. [8] analyzed the failure probability of seepage in a homogeneous dike and discussed the influence of the Cov of $\mathrm{HC}$ and the changes of flood level with a stochastic FEM. Hu et al. [9] have combined FEM and SRSM with Hermite polynomials to analyze the reliability of seepage stability of the clay core wall of Shuangjiangkou rockfill dam and studied the influence of the Cov of variables.

Great progress has been made in this field, from the hydraulic method to FEM in deterministic analysis method, from a low homogeneous dam to a high complex dam in analysis object, and from explicit formula to using FEM to construct SRSM in LSF, but reliability calculation is still in $2 \mathrm{D}$ stage. The difficulties in analyzing the bypass seepage stability of a complex reinforced earth-rockfill dam are (1) the LSF of seepage failure is highly nonlinear and cannot be expressed explicitly; (2) bypass seepage of complex reinforced earth-rockfill dam is a $3 \mathrm{D}$ problem, and large number of random variables will make reliability analysis too computationally intensive. At present, JC method is only applicable to an explicit LSF and lowly nonlinear. The stochastic FEM has a long development cycle, and it is difficult to develop a uniform stochastic FEM program to suit for different problems. The actual LSF can only be well fitted by an ordinary RSM in the vicinity of design point through adjusting response surfaces, but it can be well fitted by the SRSM with an optimal order polynomial chaos expansion in the entire space [10]. The SRSM just needs one response surface and can solve the problem with implicit LSF by using less samples than ordinary RSM, but high-order Hermite polynomials are extremely complicated [11]. Therefore, it is necessary to explore a method for the reliability analysis of 3D bypass seepage stability of earth-rockfill dam, which considers 3D seepage analysis and SRSM with simple polynomials.

The objective of this paper is to propose a practical SRSM with ordinary high-order polynomials as LSF to analyze the reliability of 3D bypass seepage stability of earth-rockfill dams. To achieve this goal, this article is organized as follows. In Section 2, a 3D seepage FEM is introduced briefly. In Section 3, procedure of the highorder practical SRSM is discussed in detail, and two numerical examples are investigated to demonstrate the validity of the proposed method. Bantou reinforced dam is taken as an example in Section 4; reliability analysis for bypass seepage stability is calculated based on the upper water level and $5 \mathrm{HCs}$ of materials as random variables. The influence of the orders and Cov of random variables are also analyzed.

\section{Seepage FEM for Earth-Rockfill Dam}

The seepage model of an earth dam with free surface and seepage surface is shown in Figure 1. The dam body can be divided into a saturation zone $\Omega_{1}$ and an unsaturated zone $\Omega_{2}$. According to Darcy's law, the waterhead at any point can be expressed as follows:

$$
\frac{\partial}{\partial x}\left(k_{x} \frac{\partial h}{\partial x}\right)+\frac{\partial}{\partial y}\left(k_{y} \frac{\partial h}{\partial y}\right)+\frac{\partial}{\partial z}\left(k_{z} \frac{\partial h}{\partial z}\right)=0,
$$

where $h$ is waterhead and $k_{x}, k_{v}$, and $k_{z}$ are the HCs in $x, y$, and $z$ directions. The corresponding boundaries of formula (1) are listed as follows:

(1) $A B$ and $C D$ are the Dirichlet boundaries of waterhead. $h_{A B}=H_{1}$ and $h_{C D}=H_{2}$.

(2) $B C$ is the Neumann boundary of flow; the flow $q_{B C}=0$

(3) $A E$ is the free surface of seepage, the interface of $\Omega_{1}$ and $\Omega_{2}$. The water level on the surface is equal to the piezometric waterhead $z$. If there is no flow exchange between the two regions, $h_{A E}=z$ and $v_{A E}=0$.

(4) $D E$ is the seepage surface. Flow outflow from the ED surface, that is, $h_{D E}=z$ and $v_{D E} \leq 0$.

The solution of equation (1) is equivalent to the minimum value of the following formula:

$$
I[h(x, y)]=\iiint_{\Omega} \frac{1}{2}\left[k_{x}\left(\frac{\partial h}{\partial x}\right)^{2}+k_{y}\left(\frac{\partial h}{\partial y}\right)^{2}+k_{z}\left(\frac{\partial h}{\partial z}\right)^{2}\right] \mathrm{d} x \mathrm{~d} y \mathrm{~d} z .
$$

Equation (2) is derived by the node waterhead in the seepage area. $[K]^{e}\{h\}^{e}=0$ can be obtained. Functional differential is conducted on all elements; multiply the conduction matrix $[K]^{e}$ and the node waterhead $\{h\}^{e}$, moving the nodes with the given waterhead to the right of the equation, formula (3) can be obtained

$$
[K]\{h\}=\{Q\},
$$

where $[K]$ is conduction matrix, $\{h\}$ is waterhead, and $\{Q\}$ is the initial flow. Since formula (3) was solved by Neumann in 1973, there have been several constant mesh methods, such as initial flow method [12], improved method of nodal virtual flux [13], Signorini variational inequality method [14], and equivalent nodal flow method [15].

If $\{h\}$ at any FEM nodes is solved, the seepage field will be determined. The total seepage gradient at any point can be defined as

$$
J=\sqrt{\left(-\frac{\partial h}{\partial x}\right)^{2}+\left(-\frac{\partial h}{\partial y}\right)^{2}+\left(-\frac{\partial h}{\partial z}\right)^{2}}
$$

\section{High-Order Practical SRSM}

The SRSM was first proposed in the study of uncertain problem in environmental and biological system by Isukapalli et al. [16] in 1998. The SRSM combining a traditional deterministic method and the uncertainty method not only overcomes the difficulty that the LSF cannot be expressed explicitly but also greatly decreases the amount of response calculation of samples compared with the traditional direct MCS. An important procedure with the SRSM is to choose the RSP. The SRSM with Chebyshev polynomials to 


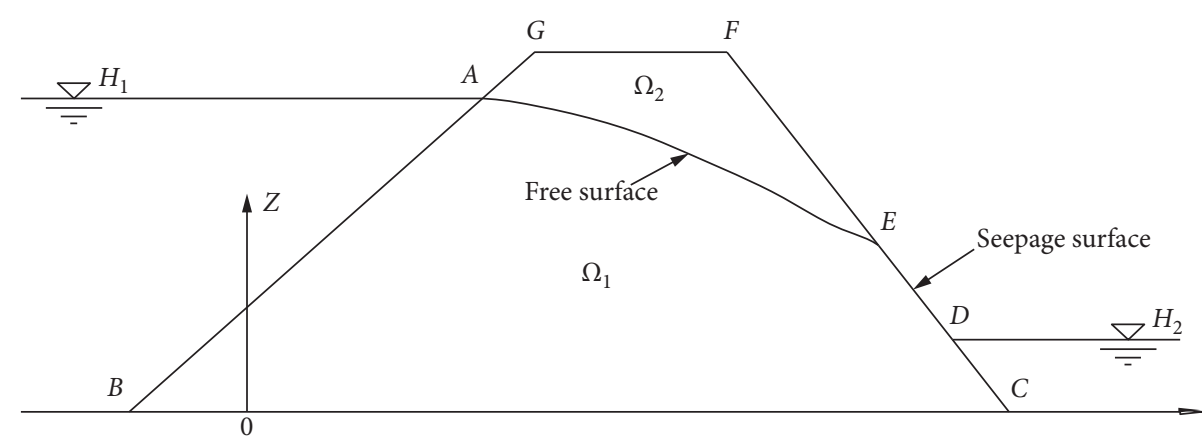

Figure 1: Seepage model with free surface and seepage surface.

determine variable orders was proposed by Gavin and Yau [17], and the SRSM with Hermite polynomials was proposed by Jiang et al. [11]. In this paper, ordinary high-order polynomials with mixed terms in equation (5) are used as the LSF surrogate model. The algorithm of the proposed method is called the high-order practical SRSM.

$$
\overline{g(x)}=a+\sum_{i=1}^{n} \sum_{p=1}^{k_{i}} b_{i p} x_{i}^{p}+\sum_{q=1}^{m} c_{q} \prod_{i=1}^{n} x_{i}^{p_{i q}},
$$

where $a$ is a constant; $\sum_{p=1}^{k_{i}} b_{i p} x_{i}^{p}$ is an independent term; and $\prod_{i=1}^{n} x_{i}^{p_{i q}}$ is the possible mixed term, where the total number of random variables $(n)$, the polynomial order $\left(k_{i}\right)$, and the coefficients $\left(b_{i p}\right)$ correspond to independent terms involving only one variable; the coefficient $c_{q}$ corresponds to mixed terms, involving the product of two important random variables, the total number of mixed terms $(m)$, and the order of a random variable in a mixed term $\left(p_{i q}\right)$.

Figure 2 shows the flow chart, mainly including four stages. First was the order identification (OI) of random variables. Secondly, estimate the importance of the level of random variables and possible mixed terms. These first two stages result in the basic formulation of the RSP as a surrogate model. The specific formulation and corresponding coefficients of the RSP are determined in the third stage by stepwise regression based on OI samplings, additional samplings, and design point or mean value point. Fourthly, MCS is carried out to determine the failure probability and estimate the design point. If the number of variables or the coefficient of variation is large, the RSP needs to be updated by adding several additional samples around the design point.

3.1. Order Identification. If a variable order of polynomials is much higher than the real LSF, ill-conditioned systems of equations may be encountered [18]. So, order identification is the base of the accuracy of the SRSM. If univariate fitting is taken, the steps of order identification of the variable are listed as follows:

Step 1.OI sample arrangement. Variable $x_{i}$ is set to $\left[\mu_{i}-f \sigma_{i}, \mu_{i}+f \sigma_{i}\right]$, where $\mu_{i}$ is a vector of the mean value of $x_{i}, \sigma_{i}$ is a vector containing the standard deviation of $x_{i}$, and interpolation coefficient $f$ is set to the value from 1 to 3 . All other variables,

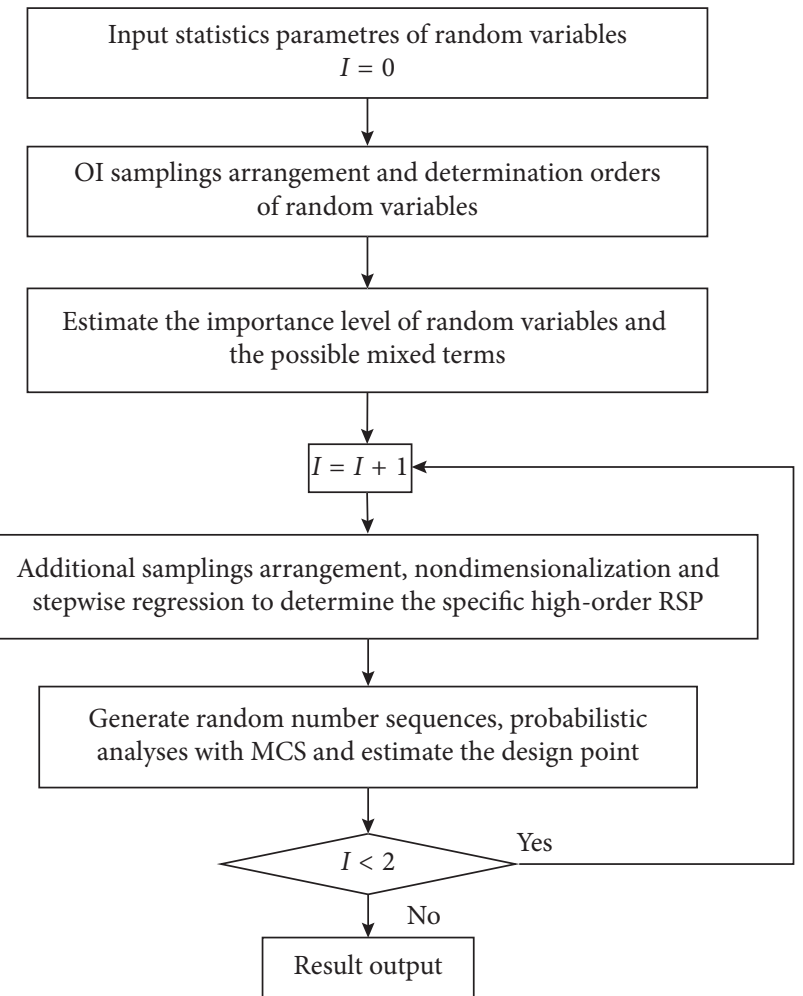

FIGURE 2: Flow chart of the high-order practical SRSM.

$x_{1}, \ldots, x_{i-1}, x_{i+1}, \ldots, x_{n}$, are set to their means $\left(\mu_{1}, \ldots, \mu_{i-1}, \mu_{i+1}, \ldots, \mu_{n}\right)$.

Step 2. Response calculation of the OI samples, $g_{i}=g\left(\mu_{1}, \ldots, \mu_{i-1}, x_{i}, \mu_{i+1}, \ldots, \mu_{n}\right)$.

Step 3. Spline curve fitting taken as the real values based on variable $x_{i}$ and corresponding $g_{i}$.

Step 4. Polynomial fitting without mixed terms based on variable $x_{i}$ and corresponding $g_{i}$ can be approximated in equation (6), and the multiple correlation coefficient $R_{1}^{2}$ can be obtained.

$$
\overline{g_{i}}=a_{0}+a_{1} x_{i}+a_{2} x_{i}^{2}+\cdots+a_{n} x_{i}^{n}
$$

Step 5. Calculate the difference $R_{j}$ between spline curve and polynomials by equation (7) from first order to the 
sixth order. $\left(x_{i}^{\prime}, y_{i}^{\prime}\right)$ and $\left(x_{i}^{\prime \prime}, y_{i}^{\prime \prime}\right)$ are the scatter points in spline curve and polynomials, respectively.

$$
\begin{aligned}
& R_{j}=\sum_{i=1}^{100} \sqrt{\left(x_{i}^{\prime}-x_{i}^{\prime \prime}\right)^{2}+\left(y_{i}^{\prime}-y_{i}^{\prime \prime}\right)^{2}} \\
& j=1,2, \ldots, 6 .
\end{aligned}
$$

Step 6. Determine the highest order of $x_{i}$. To avoid the Runge phenomenon, the order with the minimum $R_{j}$ is taken as the highest order of variable $x_{i}$. Meanwhile, to decrease the number of samplings, the orders $1 \sim \mathrm{n}$ with the multiple correlation coefficient $R_{1}^{2}$ greater than 0.995 for the first time, the order $n$ can also be taken as the highest order of variable $x_{i}$, for $R_{1}^{2}$ in polynomial fitting represents the accuracy of the fitting of the scatter, and 0.995 is in a high level. Choose the small one as the highest order of variable $x_{i}$ according to the two criterions.

Step 7.Determine the other possible order of $x_{i}$. If $n$ is the highest order of $x_{i}$, the terms $x_{i}, x_{i}^{2}, \ldots, x_{i}^{n-1}$ may not simultaneously exist in equation (6). Multiple correlation coefficient $R_{2}^{2}$ of the remaining terms can be used to exclude the unimportant order of $x_{i}$. If $R_{2}^{2} / R_{1}^{2}>0.9995$, the remaining terms are the possible orders.

The orders of the variable determined by the seven steps may exist in independent terms, mixed terms, or in both. Generally, RSP includes several independent terms and mixed terms.

\subsection{Important Level Estimation and Possible Mixed Terms of} Variables. The contribution of mixed terms to the LSF is related to the importance level of variable. On one hand, inappropriate mixed terms may lead to incorrect estimation of failure probability. On the other hand, the more the mixed terms, the larger the response calculation. Therefore, only the mixed terms of the important variables need to be considered. The importance of variables is highly correlated with the variable contribution to the uncertainty of response. The variance $\sigma_{g_{i}}^{2}$ of the response of a single variable can be obtained based on equation (6). The importance level can be defined as follows:

$$
Q_{i}=\frac{\sigma_{g_{i}}^{2}}{\sum_{i=1}^{n} \sigma_{g_{i}}^{2}} \times 100 \% .
$$

If $Q_{i}$ is less than a critical value, such as $5 \%$, the corresponding variable $x_{i}$ can be considered as an nonessential one, which can be excluded in the mixed terms to reduce the number of the samples required for polynomial fitting.

To explore the mixed terms of RSP, Gavin and Yau [17] had proposed two criteria: (1) the power of a variable in a mixed term should not be larger than the estimated order of the variable alone; (2) the total order of the mixed term should not be larger than the highest-order term. Besides, the regression additional criterion proposed by $\mathrm{Li}$ [19] is just to consider the mixed terms including the important variables. However, the Gavin-Yau criteria two cannot determine the RSP with the only mixed term, for example, $g=x y z$. The highest order of variables is 1 , but the total order of the mixed term is 3 . So, it can be changed to the power of a variable in the mixed term which should be less than the highest order of this variable except the first order, improved Gavin-Yau criterion two.

Once the basic formulation of RSP has been estimated, the specific form and corresponding coefficients of RSP can be determined by stepwise regression. Polynomial fitting requires samples uniform and representative in the solution domain, and the number of samples is best chosen to be twice that of polynomial coefficients [19]. This paper uses orthogonal table to arrange additional samples, for its balanced dispersion and neat comparable characteristics in the samples arranged.

\subsection{Additional Samples Arrangement and Stepwise Regression} of High-Order RSP. OI samples are just located in the horizontal line and vertical line, and there are no samples in each quadrant consisting of horizontal and vertical lines (shown in Figure 3). An orthogonal table will be used to arrange the additional samples to fill the blank. Every orthogonal table has two characteristics: different numbers appear equal times in each column, and the numbers in any two columns are arranged in a balanced dispersion situation. Take the $L_{4}\left(2^{3}\right)$ orthogonal table (Table 1) as an example; there are four combinations in any two columns: $(1,1),(1,2)$, $(2,1)$, and $(2,2)$. Each combination appears at the same frequency. Therefore, there is strong representation in the samples arranged by an orthogonal table.

An orthogonal table is selected according to the principle that the maximum factor is slightly great than or equal to the number of variables. Take a 2-level orthogonal table as an example; each row of the table can be taken $n$ times in arrangement of additional samples according to the mean point (or the design point $X_{d}$ ) and standard deviation $\sigma_{x}$. If there are just two random variables, $L_{4}\left(2^{3}\right)$ orthogonal table will be chosen. Columns 1 and 2 in Table 1 are used to set values for variable one and two, respectively. When the number is 1 , the corresponding variable is set as $X_{d}-f \sigma_{x}$; when the number is 2, the corresponding variable is set as $X_{d}+f \sigma_{x}$. Each row of the table can achieve one sample, so there are 4 samples marked black $\times$ in Figure 3(a). If the interpolation coefficient $f$ ranged from 1 to 3 and the orthogonal table is used 3 times, the corresponding variable can be set as $X_{d}-\sigma_{x}, X_{d}-2 \sigma_{x}$, and $X_{d}-3 \sigma_{x}$ when the number is 1 and $X_{d}+\sigma_{x}, X_{d}+2 \sigma_{x}$, and $X_{d}+3 \sigma_{x}$ when the number is 2 . There are 12 samples marked the sign $\times$ (shown in Figure 3(a)).

Latin hypercube sampling (LHS) method is a popular sampling method. Compared with the LHS method, there are two advantages in the additional samples with the orthogonal table method (Figure 3(b)): (1) more additional samples are located at the edge; (2) there is orthogonality characteristic in the additional samples with the orthogonal table method, and the number of additional samples is equal in each quadrant consisting of horizontal and vertical lines. 


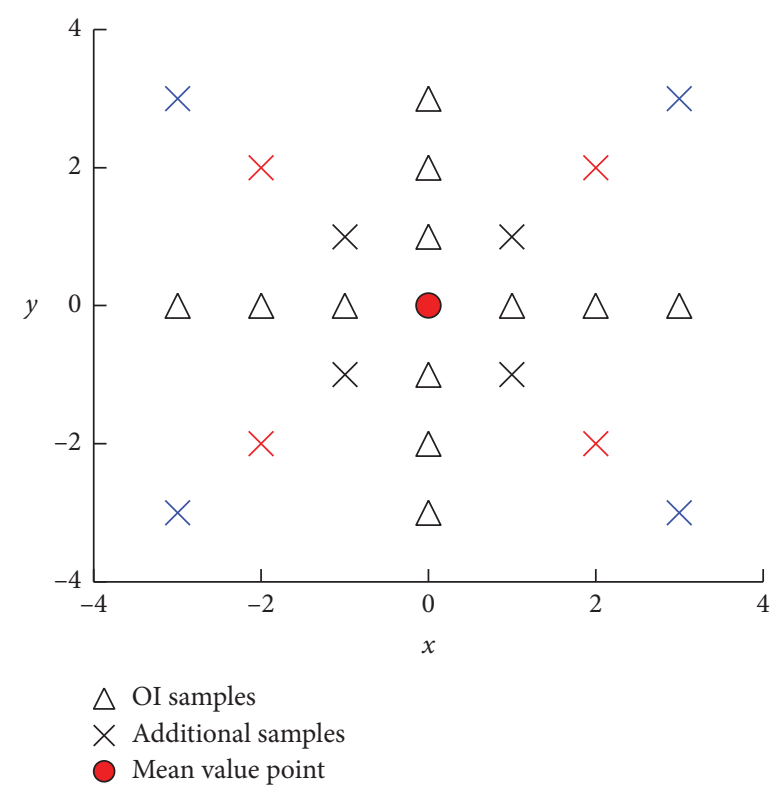

(a)

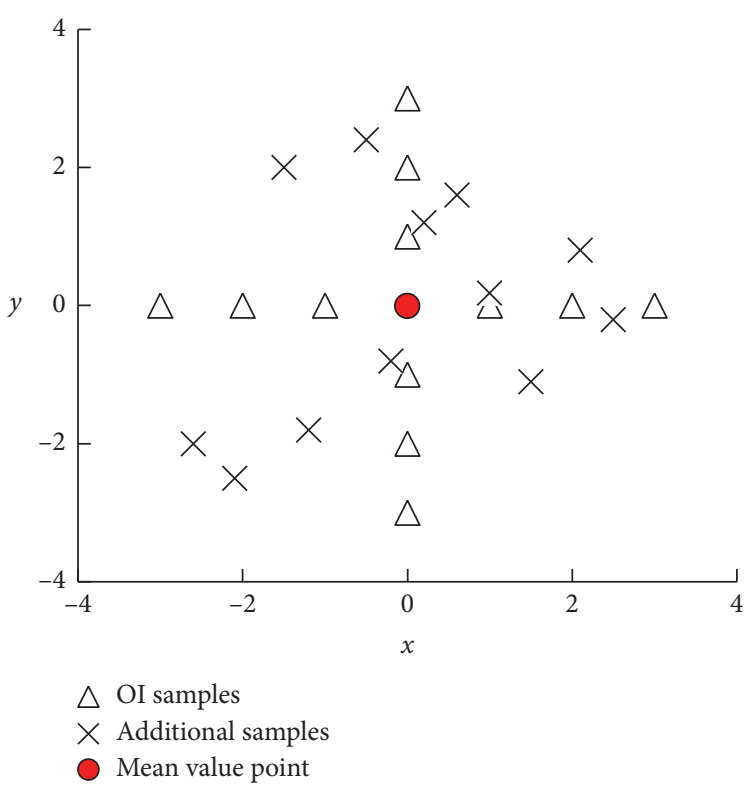

(b)

FIgURE 3: Diagram of samples. (a) Orthogonal table method. (b) Latin hypercube sampling method.

TABLE 1: $L_{4}\left(2^{3}\right)$ orthogonal table.

\begin{tabular}{lccc}
\hline Test number & Column 1 & Column 2 & Column 3 \\
\hline 1 & 1 & 1 & 1 \\
2 & 1 & 2 & 2 \\
3 & 2 & 1 & 2 \\
4 & 2 & 2 & 1 \\
\hline
\end{tabular}

The specific formulation and corresponding coefficients of RSP can be determined by stepwise regression based on OI samplings, additional samples, design point, and these corresponding responses calculated. The true LSF $g(x)$ is surrogated with an ordinary polynomial in equation (5), and the least squares method is used to determine the unknown coefficients. There are three steps to determine the specific formulation of the RSP:

Step 1. The first step is the nondimensionalization of variables and responses to avoid the occurrence of "large numbers to eat decimals" during the polynomial fitting. Assuming that $x_{i}$ and $x_{i}^{\prime}$ are the values before and after nondimensionalization, a linear dimensionless method is defined as follows:

$$
x_{i}^{\prime}=\frac{x_{i}}{\max x_{i}} .
$$

Step 2. The mix terms are added one by one to conduct regression analysis based on all possible independent terms estimated in Section 3.1. If a possible mix item is added and the fitting multiple correlation coefficient $R^{2}$ becomes larger, the new mixed term is kept. If $R^{2}$ is smaller or unchanged, exclude the new mixed item.

Step 3. The possible independent items are subtracted one by one to conduct regression analysis based on all possible independent items and the determined mix terms. If an independent term is subtracted and $R^{2}$ becomes larger or almost unchanged, the independent term is excluded. If $R^{2}$ is smaller, keep the independent term.

3.4. MCS and Design Point Estimation. The true LSF will be replaced by the RSP determined. When solving the failure probability with MCS, N random sequences will be generated according to the distribution characteristics of variables. The sampling method for the random number sequences of the related multidimensional normal distribution has been introduced in many references. If random variables are nonnormal distribution, the correlation coefficient is not consistent after equivalent normalization transformation. The correlation coefficient conversion of nonnormal random variable is commonly transformed with the Nataf method or the Copula function method. To explore the change of correlation coefficient, some empirical formulations with the Nataf method have been established $[20,21]$. A two-step numerical integration method has been proposed by Wen et al. [22] to overcome the nonconvergence in the numerical calculation of the Nataf method.

After the random number sequences are generated, by substituting them into RSP and counting the number of samples $N_{f}$ in the failure region, the failure probability is expressed as

$$
p_{f}=\frac{N_{f}}{N} .
$$

As for some events with a small probability in engineering, the total number of samples $\mathrm{N}$ should not be less than $100 / P_{f}$. In the reliability analysis, the design point has two characteristics: (1) the design point is on the failure 
boundary; that is, the LSF value of the point is 0 ; (2) the design point is with maximum failure probability on the failure boundary. According to characteristic one, $N$ samples in the MCS are sorted on the basis of the absolute value of the LSF. If $\mathrm{N}$ is greater than $10^{\wedge} 5,500$ small absolute values can be taken as on the LSF. According to the characteristic two, the design point is located in the dense area of the failure boundary. The distance between the coordinate origin and 500 samples in the standard normal space is calculated, respectively, and the density curve of the distance can be obtained. Then, the group with maximum density is chosen, and the averages of this group's variable are taken as the design point.

Above all, practical SRSM is just one initial response surface or one updated response surface, which is fitted based on OI samples, additional samples, and corresponding response calculation. Besides, practical SRSM adopts a nonintrusive form; not only the reliability analysis and the response calculation can be conducted separately but also the response of samples can be calculated on different computers or different cores of a machine.

3.5. Numerical Examples. Two numerical examples are used to illustrate the efficiency and accuracy of the high-order practical SRSM.

Example 1 (see [23]). The third-order nonlinear function $g(x, y)=x^{3}+x^{2} y+y^{3}-18, x \sim N\left(10,5^{2}\right), y \sim N\left(9.9,5^{2}\right)$ and $\rho_{x y}=0.1$; estimate the failure probability.

Interpolation coefficient was assigned from 1 to $3 ; 12 \mathrm{OI}$ samples and corresponding responses are listed in Table 2. Figure 4 shows the relationship between single variable and these corresponding responses; the highest order of variable $x$ and $y$ is 3 for both, and the multiple correlation coefficient $R_{1}^{2}$ is 1.00 . Table 3 shows the process for determining the other possible orders of variables $x$ and $y$. When $x$ is subtracted to fit polynomials, the multiple correlation coefficient $R_{2}^{2}$ of the remaining terms $x^{2}$ and $x^{3}$ is unchanged. If $x^{2}$ or $x$ and $x^{2}$ subtracted at the same time, $R_{2}^{2} / R_{1}^{2}$ will be less than 0.9995 , so the first order of $x$ is an unimportant order and can be excluded. With the same method, the term $y^{2}$ can also be omitted. Therefore, there are 4 possible independent terms $x^{2}, x^{3}, y$, and $y^{3}$ and only one possible mixed term $x^{2} y$ according to the improved Gavin-Yau criteria two. 5 terms and the constant result in basic formulation of RSP.

Use the $L_{4}\left(2^{3}\right)$ orthogonal table 2 times to arrange 8 additional samples and conduct nondimensionalization of the 21 samples (Table 2) by equation (9); the stepwise regression process for determining the specific mixed terms and independent items based on the 21 samples is listed in Table 4 . When the 4 independent terms are considered as LSF, $R^{2}$ is 0.9820 . If the mixed term $x^{2} y$ is added, $R^{2}$ is increasing to 1.00 , the mixed term will be kept. If $x^{2}$ or $y$ is subtracted, $R^{2}$ are all unchanged. If $x^{3}$ or $y^{3}$ is subtracted, $R^{2}$ are decreased, so the independent terms $x^{3}$ and $y^{3}$ should be kept and $x^{2}$ and $y$ can be omitted. $g(x, y)=-0.00075+0.65589 x^{3}+0.64805 y^{3}+0.65326 x^{2} y$ is obtained, and the 3 terms and the constant are perfectly determined.

Taking the RSP as LSF, generate $1 \times 10^{7}$ random number sequences with correlation coefficient 0.1 with the Nataf method. Table 5 summarizes the results with the other methods; the failure probability of practical SRSM is $8.112 \times 10^{-3}$, almost consistent with the reference solution $\left(8.110 \times 10^{-3}\right)$ in [24], and the number of calling functions is close to that of the Hermite SRSM. The 500 samples with the smallest absolute value of the LSF are plotted in Figure 5, and the estimated design point is $(1.7068,1.9640)$. It can be seen that the 500 samples are all located on the true LSF, and the estimated design point is located near the bump. These results indicate that the high-order practical SRSM evaluates the failure probability accuracy.

Example 2 (see [24]). $g(x)=0.16(x-1)^{3}-y+4-0.04$ $\cos (x y)$ is a third-order highly nonlinear LSF; the random variables are subject to independent standard normal distributions, and the failure probability is calculated.

$f$ is set from 1 to 2 , and 8 OI samples can be arranged. The highest order of variable $x$ and $y$ is 3 and 1 with the method in Section 3.1. If $x, x^{2}$, and the two terms are subtracted, the $R_{2}^{2} / R_{1}^{2}$ are $0.9905,0.8368$, and 0.8273 , respectively. So, the three terms cannot be excluded. Therefore, there are 4 possible independent terms $x, x^{2}, x^{3}$, and $y$ and two possible mixed terms $x y$ and $x^{2} y$ according to the improved Gavin-Yau criteria two. 6 terms and the constant result in basic formulation of RSP.

Use the $L_{4}\left(2^{3}\right)$ orthogonal table 2 times to arrange 8 additional samples and conduct the nondimensionalization. The stepwise regression for determining the specific mixed terms and independent items is listed in Table 6. When the 4 independent terms are considered as LSF, $R^{2}$ is 0.9999 . If the mixed term $x y$ or $x^{2} y$ is added one by one, $R^{2}$ is still unchanged. So, the two mixed terms can be omitted. If the possible independent items $x, x^{2}, x^{3}, y, R^{2}$ substracted one by one, R2 is decreased to $0.9863,0.8041,0.9456$, and 0.7374 , respectively, so all the independent terms should be kept. $g(x, y)=0.56082+0.21176 x-0.44117 y-0.63195 x^{2}$ $+0.63529 x^{3}$ can be obtained. Taking the polynomials as LSF, generating $2 \times 10^{5}$ random number sequences, the failure probability is $3.3405 \times 10^{-2}$ with MCS. For comparison, Table 7 summarizes the results with the other methods; note that the failure probability of practical SRSM is the closest to that of Hermite SRSM, and the number of calling functions is 17, which is slightly larger than that of the Hermite SRSM and substantially smaller than that of Gavin-Yau SRSM. The estimated design point plotted in Figure 6 is $(-1.7968$, $0.4805)$. These results indicate that the high-order practical SRSM evaluates the failure probability efficiency and accuracy.

\section{Engineering Application}

4.1. Project Profile. The Bantou dam (Figures 7 and 8) is an earth-rockfill dam with a height of $50.3 \mathrm{~m}$. The crest 
TABLE 2: Samples of example one.

\begin{tabular}{|c|c|c|c|c|c|c|c|c|}
\hline Sample name & Serial number & $x$ & $y$ & $g(x, y)$ & Serial number & $x$ & $y$ & $g(x, y)$ \\
\hline \multirow{6}{*}{ OI samples } & 1 & -5 & 9.9 & 1074.8 & 7 & 10 & -5.1 & 339.3 \\
\hline & 2 & 0 & 9.9 & 952.3 & 8 & 10 & -0.1 & 972.0 \\
\hline & 3 & 5 & 9.9 & 1324.8 & 9 & 10 & 4.0 & 1446.0 \\
\hline & 4 & 15 & 9.9 & 6554.8 & 10 & 10 & 14.9 & 5779.9 \\
\hline & 5 & 20 & 9.9 & 12912.3 & 11 & 10 & 19.9 & 10852.6 \\
\hline & 6 & 25 & 9.9 & 22764.8 & 12 & 10 & 24.9 & 18910.2 \\
\hline \multirow{4}{*}{ Additional samples } & 1 & 5 & 4.9 & 347.1 & 5 & 15 & 5.0 & 4607.0 \\
\hline & 2 & 0 & -0.1 & -18.0 & 6 & 20 & 0.0 & 7982.0 \\
\hline & 3 & 5 & 14.9 & 3787.4 & 7 & 15 & 14.9 & 10017.4 \\
\hline & 4 & 0 & 19.9 & 7862.6 & 8 & 20 & 19.9 & 23822.6 \\
\hline Mean value point & 1 & 10 & 9.9 & 2942.3 & & & & \\
\hline
\end{tabular}

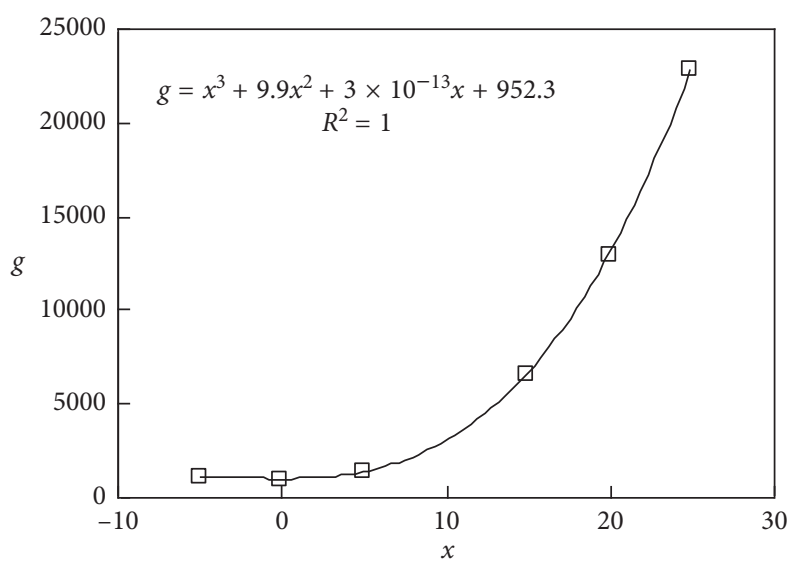

(a)



(b)

Figure 4: Order identification of random variables.

TABLE 3: Determining the possible orders of variables.

\begin{tabular}{lccc}
\hline Variable & Reserved items & $R_{2}^{2} / R_{1}^{2}$ & Keep/discard \\
\hline & $x, x^{2}, x^{3}$ & 1.0000 & Discard $x$ \\
& $x^{2}, x^{3}$ & 1.0000 & Keep $x^{2}$ \\
& $x, x^{3}$ & 0.9991 & Cannot discard $x$ and $x^{2}$ at the same time \\
& $x^{3}$ & 0.9980 & Keep $y$ \\
$y$ & $y, y^{2}, y^{3}$ & 1.0000 & Discard $y^{2}$ \\
& $y^{2}, y^{3}$ & 0.9976 & 1.0000 \\
$y, y^{3}$ & 0.9947 & Cannot discard $y$ and $y^{2}$ at the same time \\
\hline
\end{tabular}

TABLE 4: Stepwise regression for determining the mixed terms and independent terms of example one.

\begin{tabular}{|c|c|c|c|c|}
\hline Items & & Operation & $R^{2}$ & Keep/discard \\
\hline Basis terms & $x^{2}, x^{3}, y, y^{3}$ & & 0.9820 & \\
\hline Possible mixed terms & $x^{2} y$ & Add & 1.0000 & Keep \\
\hline Possible independent terms & $\begin{array}{c}x^{2} \\
x^{3} \\
y \\
y^{3}\end{array}$ & $\begin{array}{l}\text { Subtract } \\
\text { Subtract } \\
\text { Subtract } \\
\text { Subtract }\end{array}$ & $\begin{array}{l}1.0000 \\
0.9047 \\
1.0000 \\
0.8145 \\
\end{array}$ & $\begin{array}{c}\text { Discard } \\
\text { Keep } \\
\text { Discard } \\
\text { Keep }\end{array}$ \\
\hline
\end{tabular}

elevation is $312.88 \mathrm{~m}$, and the normal water level is $310.58 \mathrm{~m}$. The width of dam crest is $5.8 \mathrm{~m}$, and the length is $285 \mathrm{~m}$. The section of dam body mainly consists of filled soil and downstream rockfill; the total thickness of alluvial deposits at the riverbed and the left bank is small but is more than $20 \mathrm{~m}$ at the right bank. In 2009, the dam was reinforced, and the 
TABLe 5: Comparison of calculation result of example one.

\begin{tabular}{lcc}
\hline Method & Failure probability $\left(\times 10^{-3}\right)$ & Number of calling functions \\
\hline Hermite SRSM $(m=3)$ & 8.067 & 19 \\
High-order practical SRSM & 8.112 & 21 \\
NESSUS-MCS & 8.110 & $10^{7}$ \\
\hline
\end{tabular}

TABLE 6: Stepwise regression for determining the mixed terms and independent items of example two.

\begin{tabular}{|c|c|c|c|c|}
\hline \multicolumn{2}{|c|}{ Items } & Operation & $R^{2}$ & Keep/discard \\
\hline Basis terms & $x, x^{2}, x^{3}, y$ & & 0.9999 & \\
\hline \multirow{2}{*}{ Possible mixed terms } & $x y$ & Add & 0.9999 & $\mathrm{D}$ \\
\hline & $x^{2} y$ & Add & 0.9999 & $\mathrm{D}$ \\
\hline \multirow{4}{*}{ Possible independent terms } & $x$ & Subtract & 0.9863 & $\mathrm{~K}$ \\
\hline & $x^{2}$ & Subtract & 0.8041 & $\mathrm{~K}$ \\
\hline & $x^{3}$ & Subtract & 0.9456 & $\mathrm{~K}$ \\
\hline & $y$ & Subtract & 0.7374 & $\mathrm{~K}$ \\
\hline
\end{tabular}

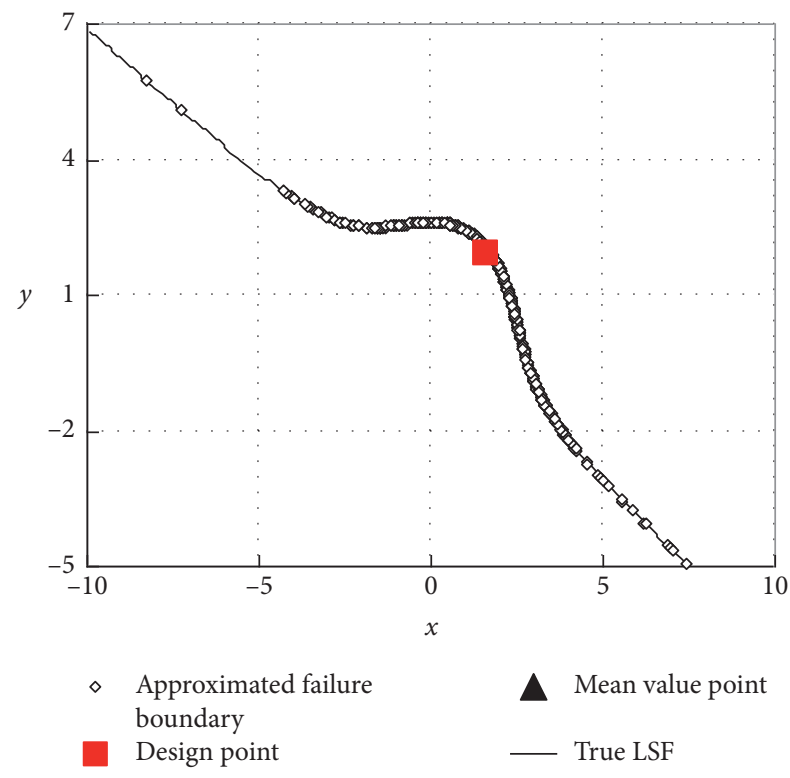

FIGURE 5: LSF of example one.

TABLE 7: Comparison of calculation result of example two.

\begin{tabular}{lcc}
\hline Method & $\begin{array}{c}\text { Failure probability } \\
\left(\times 10^{-2}\right)\end{array}$ & $\begin{array}{c}\text { Number of calling } \\
\text { functions }\end{array}$ \\
\hline $\begin{array}{l}\text { Hermite SRSM } \\
(m=5)\end{array}$ & 3.3661 & 13 \\
High-order practical & 3.3405 & 17 \\
SRSM & 3.2466 & 43 \\
Gavin-Yau SRSM & 3.2833 & $10^{6}$ \\
NESSUS-MCS & & \\
\hline
\end{tabular}

reinforcement measures include the following: (1) a new $80 \mathrm{~cm}$ thick low elastic modulus concrete cut-off wall is built on the upstream side, and the cut-off wall is directly inserted into the bedrock at the left bank and only to the bottom of the dam at the right bank; (2) curtain grouting is constructed under the dam foundation at the right bank.

The FEM model for seepage analysis (Figure 9) consists of 50,748 nodes and 49,143 elements. The statistical parameters of random variables are shown in Table 8 . The average of the

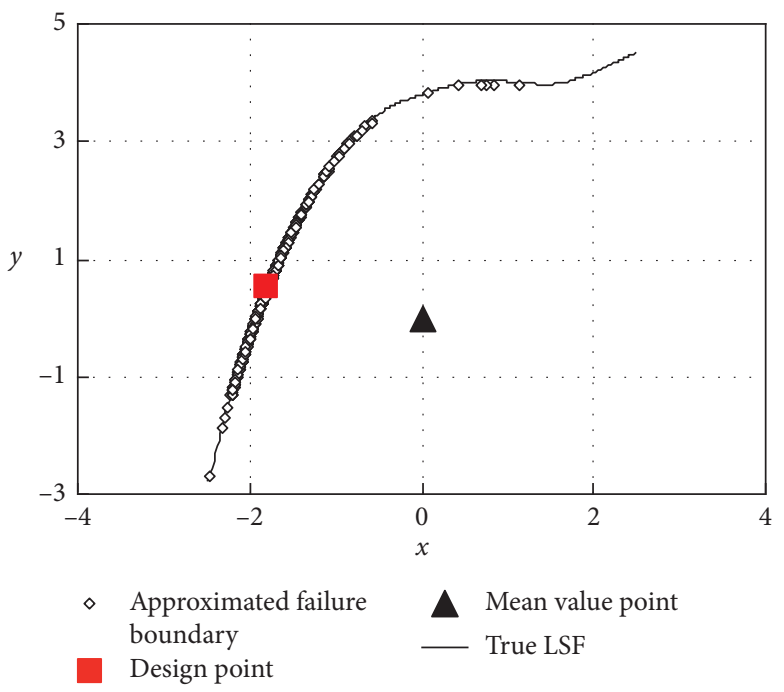

FIgURE 6: LSF of example two.

upper water level is $309.58 \mathrm{~m}$, ranging from $304 \mathrm{~m}$ to $312 \mathrm{~m}$, subject to the extremal type I distribution. The $\mathrm{HC}$ of rockfill is much larger than filled soil. The HC of curtain and cut-off wall is much smaller than that of filled soil. The HC of alluvial deposits and the HC of filled soil are in the same power. All of the $\mathrm{HC}$ are log-normal distribution.

Select the check flood condition (the upper and downstream water level are $311.99 \mathrm{~m}$ and $264.9 \mathrm{~m}$, respectively) for 3D seepage analysis. The results are shown in Figures 10 to 12 . Note that the waterhead in the downstream dam body is significantly reduced (Figure 10), but the bypass seepage is still serious (Figure 11) after the reinforcement. Figure 12 shows the seepage gradient of the downstream dam foundation. Due to the bypass seepage, the maximum seepage gradient appears at the right steep bank slope, not at the foot of the main riverbed.

4.2. Reliability Analysis of Bypass Seepage Stability. The concrete cut-off wall and curtain do the main seepage prevention of this project. Although the seepage gradient of 


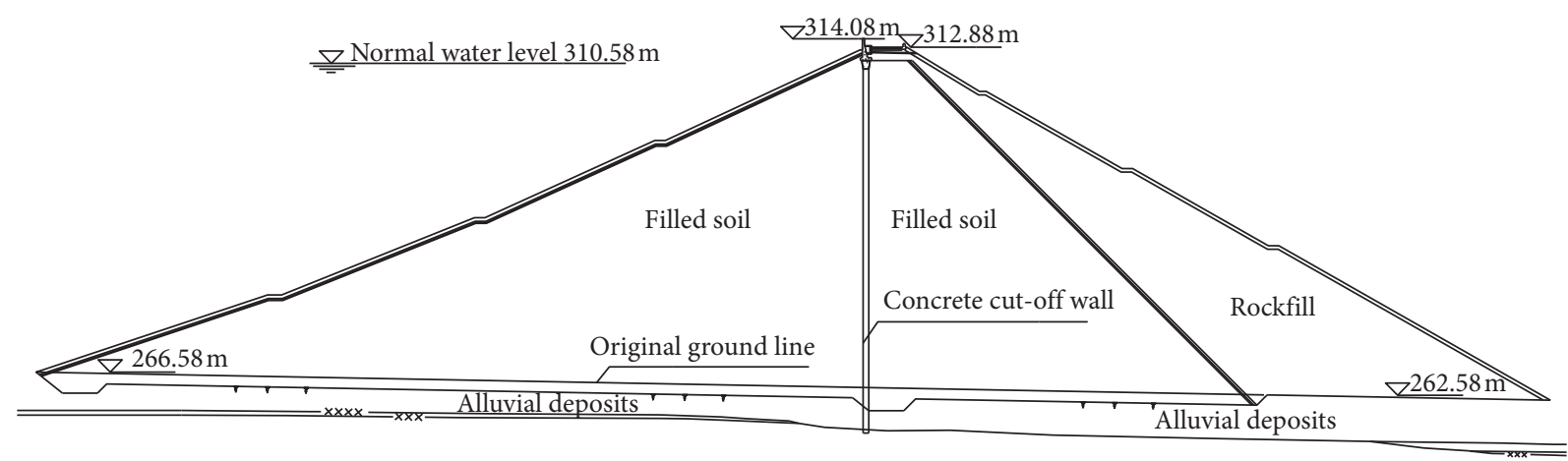

Figure 7: Typical section.

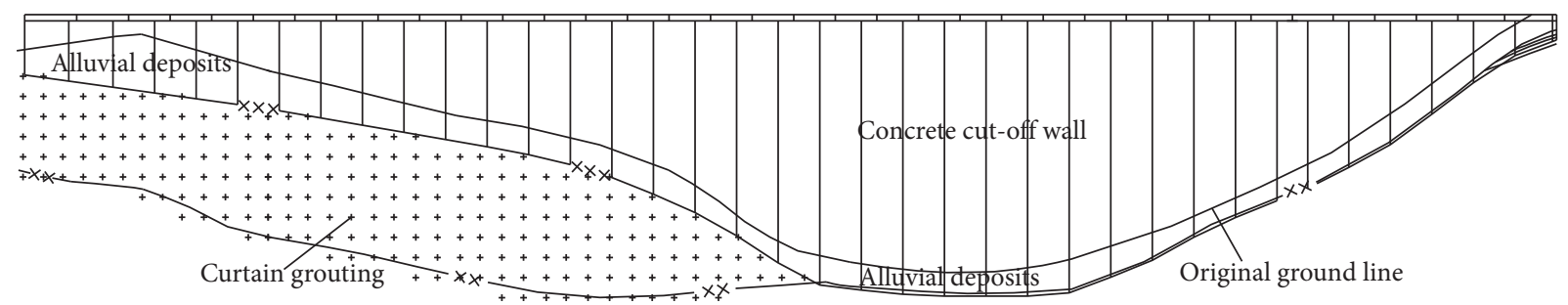

FIgURE 8: Cut-off wall and curtain grouting.

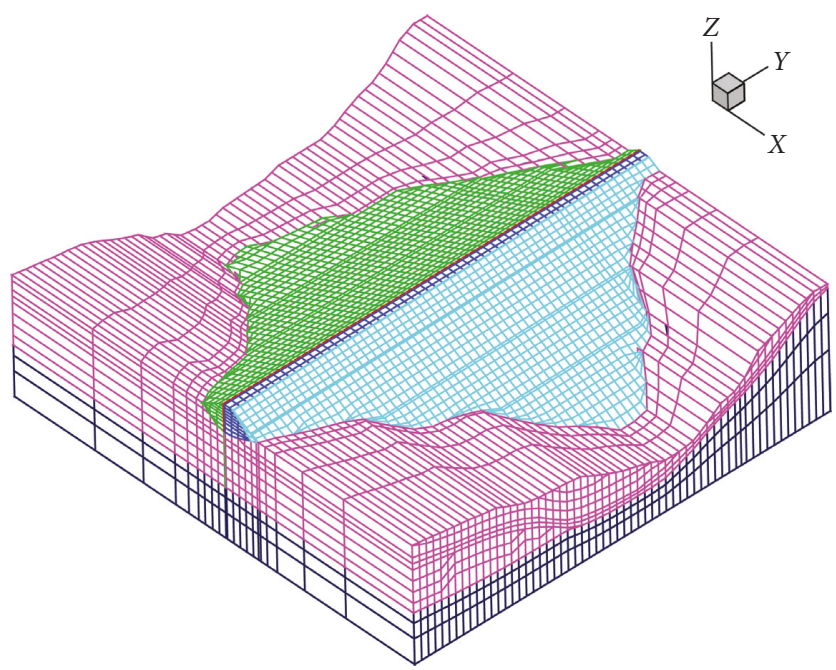

Figure 9: FEM model.

TABLE 8: Statistical parameters of random variables.

\begin{tabular}{lcccccc}
\hline \multirow{2}{*}{ Variable } & \multirow{2}{*}{ The upper water level/m } & Cut-off wall & Filled soil & Rockfill & Alluvial deposits & Curtain \\
& & & $1 \times 10^{-7}$ & $2 \times 10^{-4}$ & $2 \times 10^{-2}$ & $5 \times 10^{-4}$ \\
Mean & 309.58 & 0.06 & 0.12 & 0.16 & $5 \times 10^{-6}$ \\
Cov & 0.06 & & 0.13 & 0.09 \\
\hline
\end{tabular}

cut-off wall and curtain is large, it is much smaller than the allowable value. The seepage gradient at the bank will play a decisive role in the overall seepage stability of the dam. Therefore, the maximum seepage gradient at the right bank is used to judge the overall seepage failure of the dam. The LSF can be written as

$$
g(X)=J_{\mathrm{cr}}-J\left(X_{1}, X_{2}, \ldots, X_{n}\right)
$$

where $J$ is the maximum seepage gradient at the bank calculated by $3 \mathrm{D}$ seepage FEM; $J_{\mathrm{cr}}$ is the critical seepage gradient, equal to $0.45 ; X_{1}, X_{2}, \ldots, X_{\mathrm{n}}$ are random variables. Take the upper water level, the HC of concrete cut-off wall, 


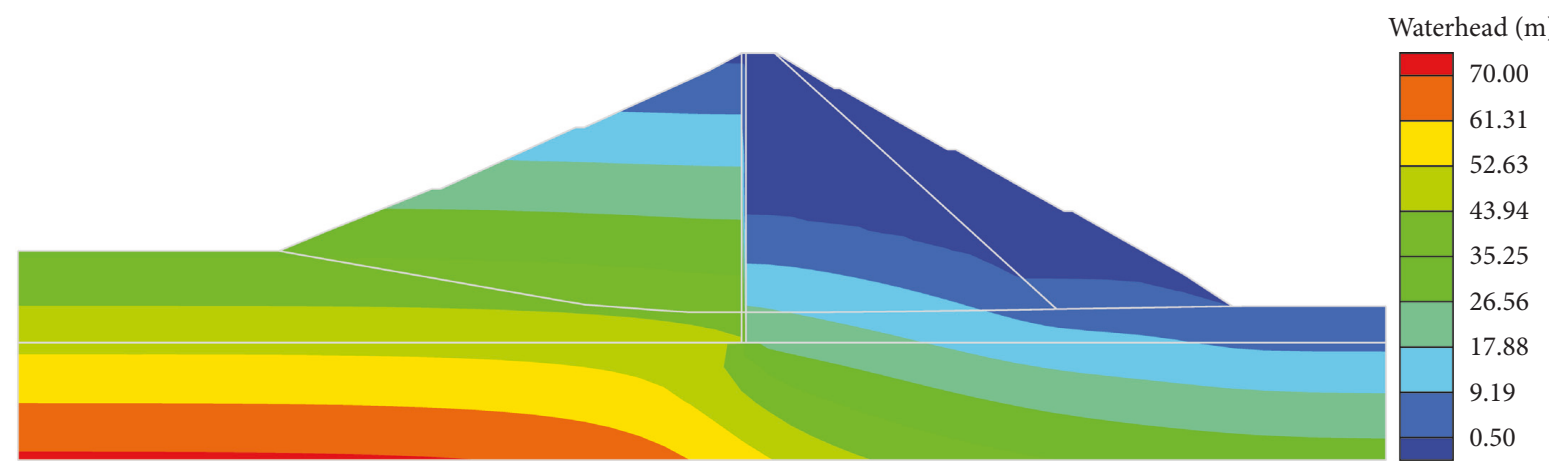

FIgURE 10: Distribution of waterhead at the largest section.
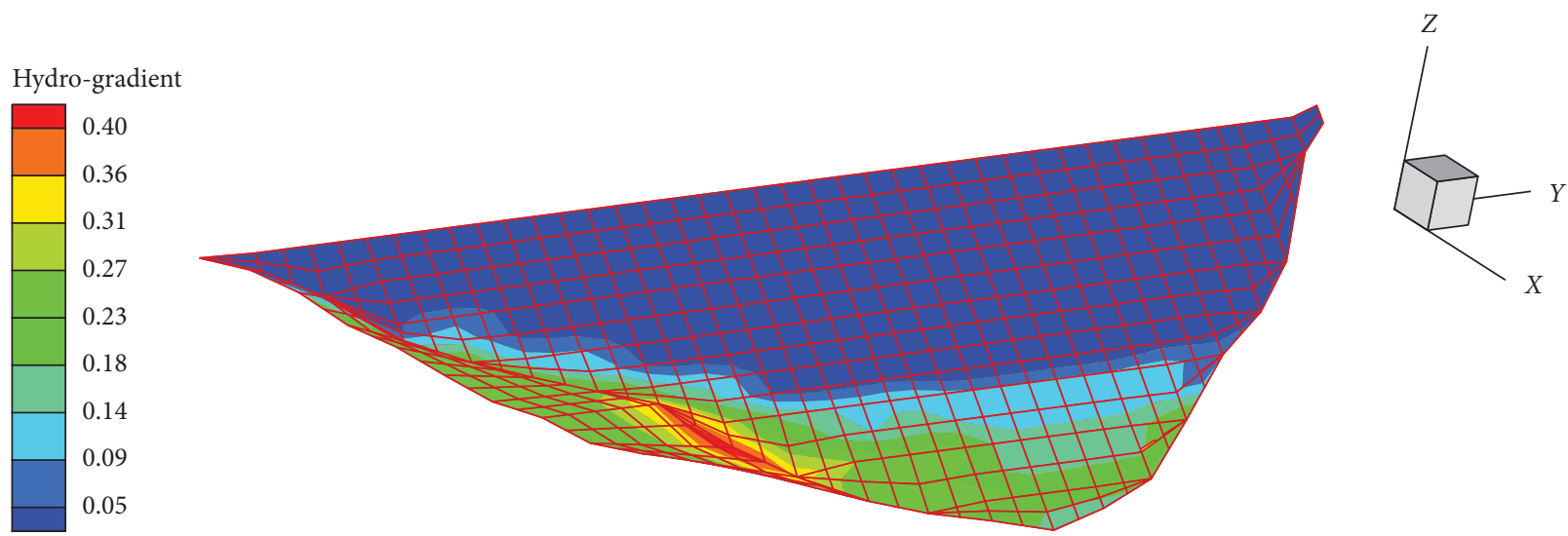

Figure 11: Distribution of seepage gradient in filled soil.

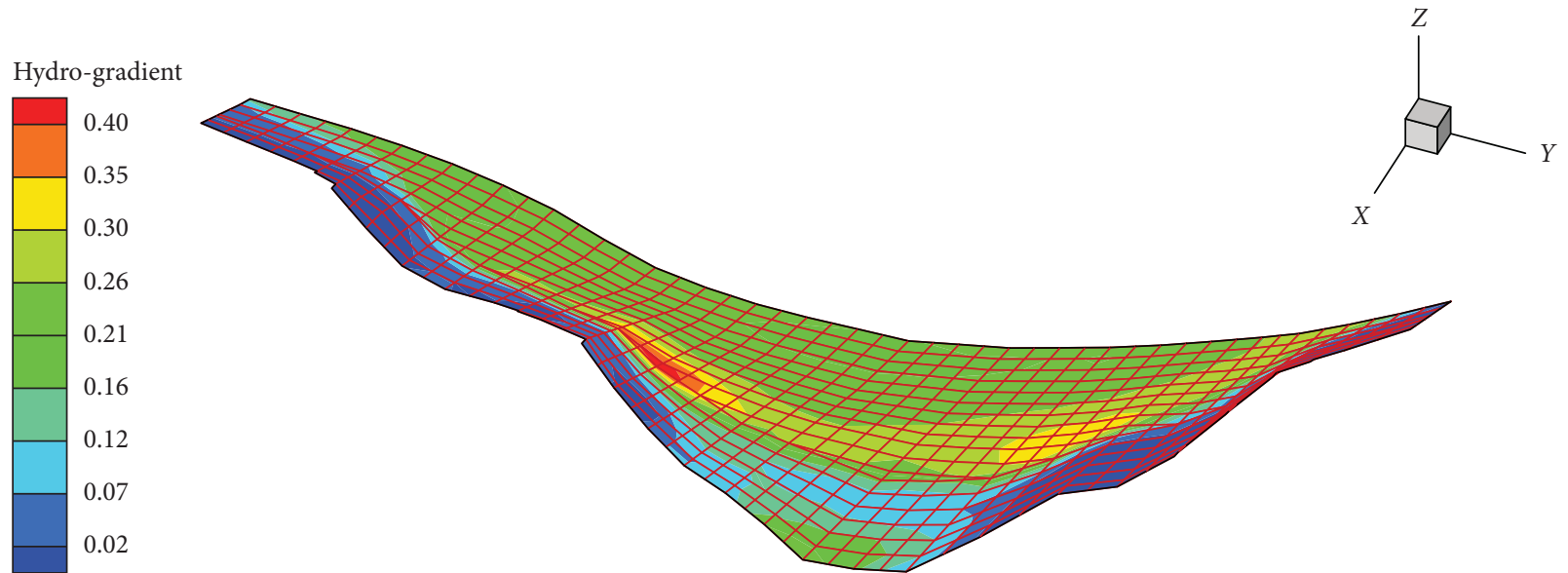

Figure 12: Distribution of seepage gradient on the downstream dam foundation.

filled soil, rockfill, alluvial deposits, and curtain as random variables.

The importance level of random variables is listed in Table 9; it can be seen that the HC of cut-off wall and rockfill are nonessential variables, since the total importance level of the two variables is less than 5\%, and the nonessential variables will be taken as constant to decrease the intensive computation. The OI of random variables is shown in Figure 13. Note that the highest order of $\mathrm{HC}$ of filled soil is 3; the $\mathrm{HC}$ of curtain is 2 and the others are all 1 . The orders lower than the highest order of each variable cannot be excluded by Step 7 in Section 3.1. There are 17 possible terms in the polynomials, including 7 independent terms, 9 mixed terms based on the three criteria, and 1 constant. Choosing the $\mathrm{L}_{8}\left(2^{6}\right)$ orthogonal table to arrange samples 3 times, adding the mean point and 24 OI samplings, there are 49 samples in total. 3D seepage program is used to calculate the seepage gradient of additional samples on different computer cores. Nondimensionalization is conducted on the samples and corresponding responses. The initial response 
TABLE 9: Importance level of random variables.

\begin{tabular}{|c|c|c|c|c|c|c|}
\hline Variables & $\begin{array}{l}\text { The upper water level } \\
\qquad X_{1}\end{array}$ & $\begin{array}{l}\text { HC of cut-off } \\
\text { wall }\end{array}$ & $\begin{array}{c}\text { HC of filled soil } \\
X_{2}\end{array}$ & $\begin{array}{l}\mathrm{HC} \text { of } \\
\text { rockfill }\end{array}$ & $\begin{array}{c}\text { HC of alluvial deposits } \\
\qquad X_{3}\end{array}$ & $\begin{array}{c}\text { HC of curtain } \\
X_{4} \\
\end{array}$ \\
\hline $\begin{array}{l}\text { Importance } \\
\text { level }\end{array}$ & 56.62 & 0.05 & 5.93 & 1.81 & 22.28 & 16.36 \\
\hline
\end{tabular}

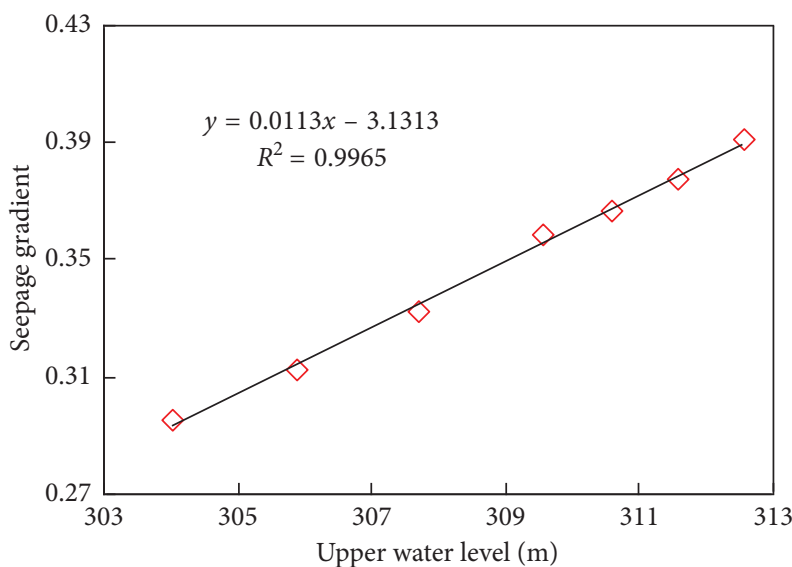

(a)

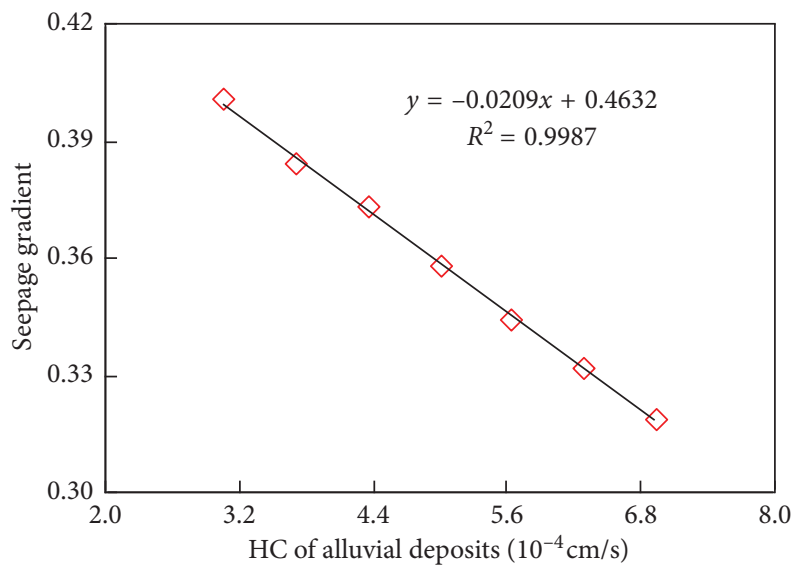

(c)

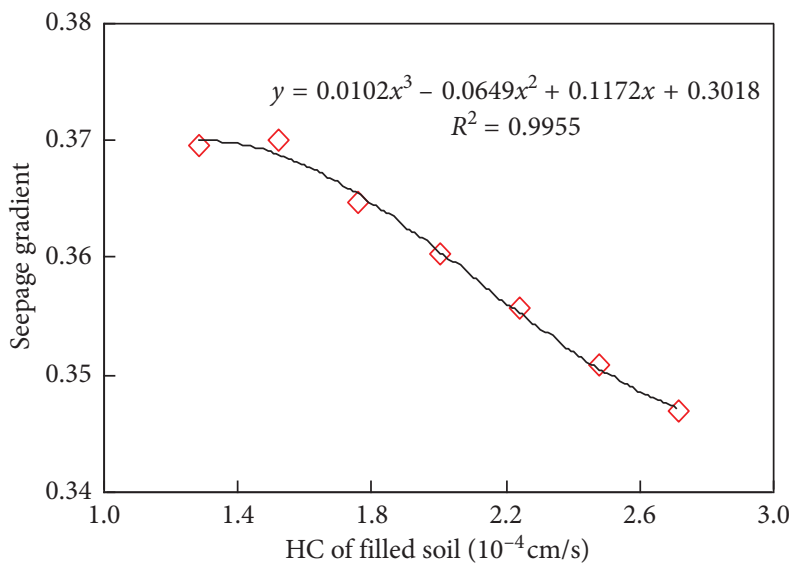

(b)

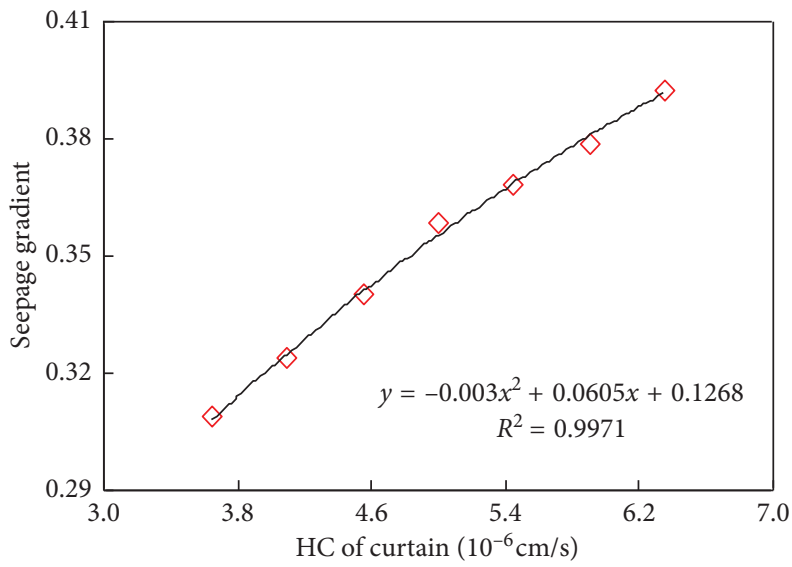

(d)

FIGURE 13: Order identification of random variables.

surface is stepwise regressed, and the initial design point is $\left(311.992,1.737 \times 10^{-4}, 3.905 \times 10^{-4}, 6.148 \times 10^{-6}\right)$. The orthogonal table was used to arrange samples one time and the interpolation coefficient was set to $0.25 ; 8$ additional samplings can be obtained; adding the design point and the previous 49 samples, the polynomials regressed is listed in formula (12), $5 \times 10^{7}$ random number sequences are generated. The failure probability is $3.680 \times 10^{-5}$, and the corresponding reliability index is 3.964 with MCS. The new design point is $\left(311.996,1.799 \times 10^{-4}, 3.744 \times 10^{-4}\right.$, $6.263 \times 10^{-6}$ ). Taking it into the $3 \mathrm{D}$ seepage program, the bypass seepage gradient is 0.4483 , similar to the critical value 0.45 . Therefore, the calculation results of this method are reliable.

$$
\begin{aligned}
g(X)= & 0.0085+0.9222 X_{1}-0.6400 X_{3}-0.3861 X_{4}^{2} \\
& +0.0242 X_{2}^{3}-0.7990 X_{1} X_{2}+0.4168 X_{1} X_{2}^{2} \\
& +0.1724 X_{1} X_{3}+0.7012 X_{1} X_{4}-0.0856 X_{2} X_{3} \\
& +0.2167 X_{2}^{2} X_{3}+0.8403 X_{2} X_{4}-0.6684 X_{2}^{2} X_{4} \\
& +0.1289 X_{3} X_{4} .
\end{aligned}
$$

Figure 14 shows the correlation between the 4 random variables and the maximum seepage gradient at the right bank. It can be seen that (1) the maximum bypass seepage gradient is highly related to the upper water level and the HC of alluvial deposits and curtain and is irrelevant to the $\mathrm{HC}$ of 


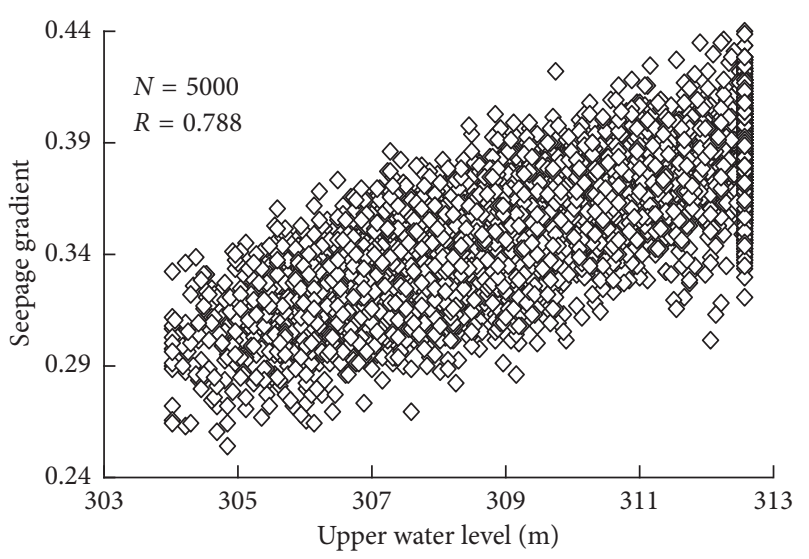

(a)

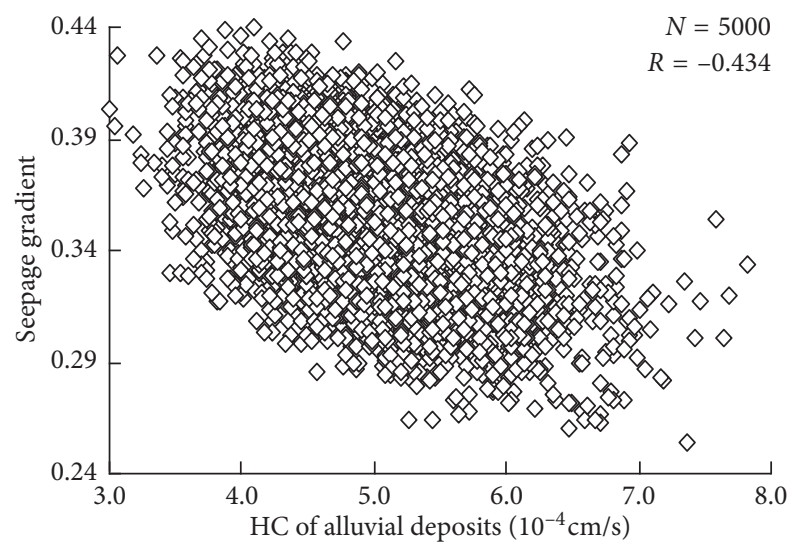

(c)

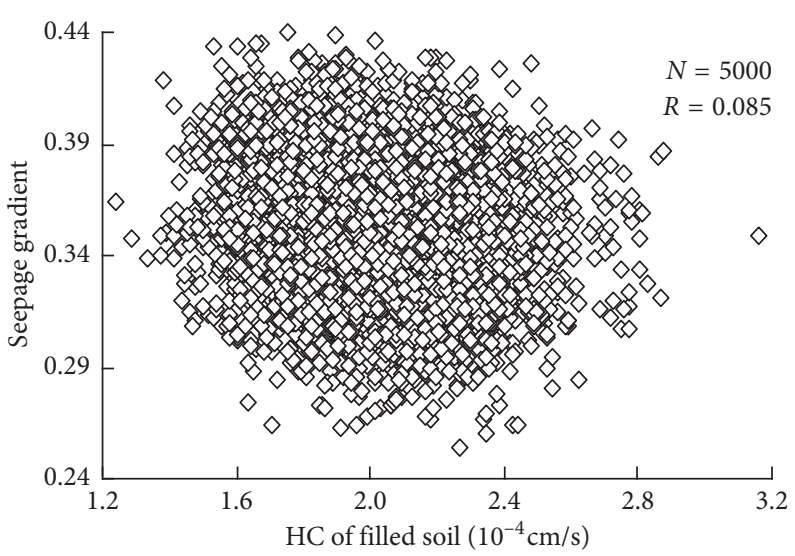

(b)

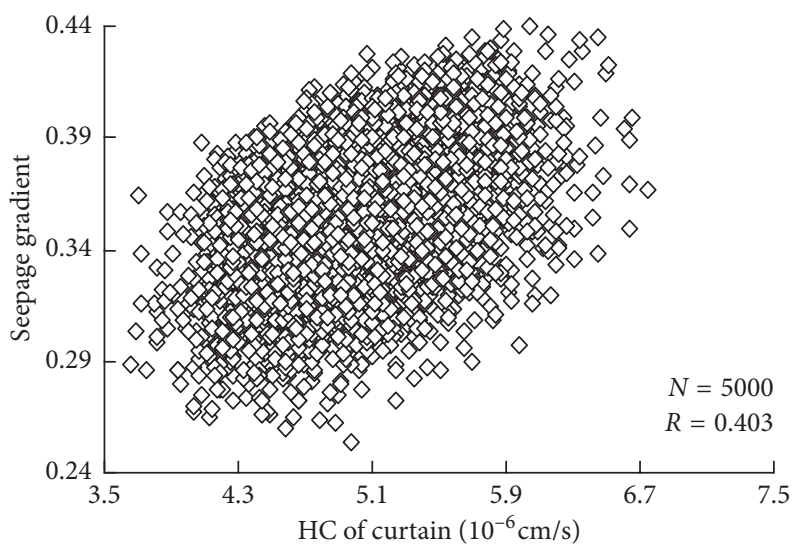

(d)

FIGURE 14: Scatter plots between the maximum seepage gradient at the right bank and random variables.

filled soil; (2) the maximum bypass seepage gradient is positively correlated with the upper water level and the HC of curtain and negatively correlated with the $\mathrm{HC}$ of alluvial deposits. The waterhead difference increases with the increase of the upper water level. The greater the $\mathrm{HC}$ of curtain is, the more the water appears at the bank slope and leads a larger seepage gradient. The larger the HC of alluvial deposits, the higher the waterhead in the downstream filled soil, and a smaller seepage gradient will be caused.

For comparison, the ordinary response surface method (RSM) is used for the reliability analysis of this project. Taking the complete cube polynomials without mixed terms as response surface, the reliability index is 3.76. 6 response surfaces and 144 samples are required, which is 2.48 times of the samples of high-order practical SRSM. These results indicate that the high-order practical SRSM is a powerful analysis method.

\subsection{Influence of the Coefficient of Variation of Random} Variables. Figure 15 shows the relationship between the failure probability of bypass seepage gradient and the upper water level and the $\mathrm{HC}$ of alluvial deposits and the $\mathrm{HC}$ of curtain. Note that the maximum bypass seepage failure probability increases more and more slowly with the increase of the Cov of the upper water level. As the Cov of HC of alluvial deposits and curtain increases, significant increases of seepage failure probability can be seen. Meanwhile, the growth rate of bypass seepage failure probability with the Cov of $\mathrm{HC}$ of curtain is larger than that of alluvial deposits. Also, the $\mathrm{HC}$ of curtain has a significant impact on the seepage gradient of the dam.

4.4. Influence of Variable Order. If the $\mathrm{HC}$ of filled soil is set from 2 nd to 4 th order, the multiple correlation coefficient $R^{2}$ of the OI curve line is $0.984,0.996$, and 0.998 , respectively, and the reliability results are listed in Table 10. The cumulative distribution function (CDF) of maximum bypass seepage gradient at the bank slope is shown in Figure 16. Note that the reliability index is getting smaller and smaller with the increasing of the order and is nearly the same when the $\mathrm{HC}$ of filled soil is from 3rd and 4th order. If the interval of bypass seepage gradient is greater than 0.34 , the three $\mathrm{CDF}$ curve lines are almost in a line. For the domain of bypass seepage gradient less than 0.34 , there is a large difference between the curve lines of 2 nd and other orders, but the 3rd and 4th order curve lines are very close.

If the HC of filled soil is set from 2nd to 3rd order, the multiple correlation coefficient $R^{2}$ of the order identification curve line is 0.992 and 0.998 , and the corresponding 


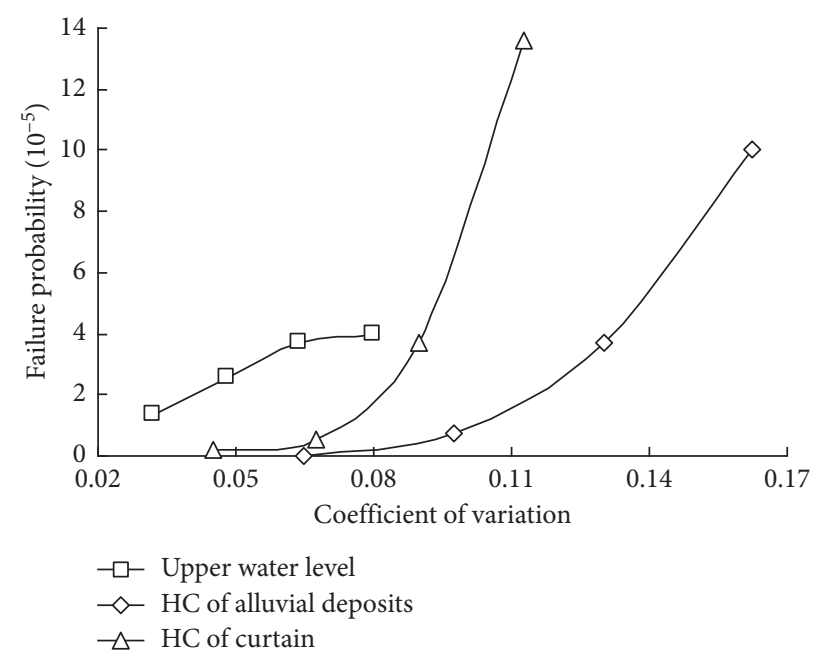

FIGURE 15: Relationship between the failure probability and the Cov of variables.

TABLE 10: Comparison table for the results of different orders of HC of filled soil with SRSM.

\begin{tabular}{lccc}
\hline Order & 2nd & 3rd & \\
\hline$R^{2}$ & 0.992 & 0.998 & 4 th \\
Reliability index & 4.085 & 3.964 & 0.998 \\
\hline
\end{tabular}

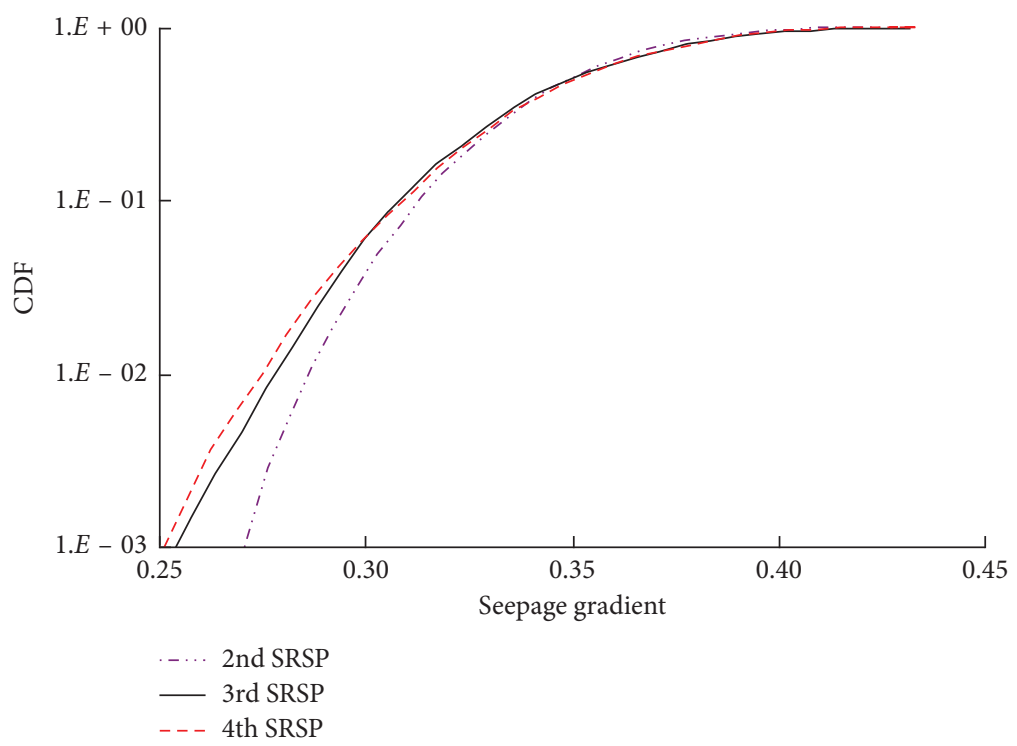

FIGURE 16: Comparison of CDF for bypass seepage gradient with maximum failure probability.

reliability results are 4.105 and 3.964 , respectively. Our results demonstrated that variable order plays a role in the accuracy of the reliability index. The larger the importance level, the larger the multiple correlation coefficient required, and the more accurate the reliability index obtained.

\section{Conclusions}

(1) A high-order polynomial with mixed terms is used as RSP. The order identification of random variables, the determination of mixed terms, and the stepwise regression of high-order polynomials are simple. The high-order practical SRSM provides a new method to estimate the failure probability of engineering problems with a certain high nonlinearity and cannot be explicitly expressed.

(2) The high-order practical SRSM adopts a nonintrusive form. The order identification samples and the additional samples arranged; the response calculation of samples can be performed on different 
computers or different cores of a machine, greatly improving the calculation efficiency. Therefore, the realization of large engineering reliability analysis can be achieved.

(3) The HC of concrete cut-off wall and the HC of rockfill are unimportant variables. The reliability index for bypass seepage stability around the Bantou dam is about 3.964, and the failure probability is $3.680 \times 10^{-5}$. The seepage failure at the bank slope is within an acceptable risk range. The maximum bypass seepage gradient has a great correlation with the $\mathrm{HC}$ of curtain, alluvial deposits, and the upper water level and has little correlation with the HC filled soil. The maximum bypass seepage gradient is positively correlated with the upper water level and the $\mathrm{HC}$ of curtain and negatively correlated with the $\mathrm{HC}$ of alluvial deposits. As the $\mathrm{Cov}$ of the $\mathrm{HC}$ of curtain increases, the bypass seepage failure probability increases dramatically.

(4) The RSP of bypass seepage can be considered as the 3rd order. As long as the reliability calculation performed using a third order or a larger order RSP, stable results can be obtained. How to achieve the sensitivity coefficient of the random variables to the seepage failure based on the high-order practical SRSM needs further research.

\section{Data Availability}

All data used to support the findings of this study are available from the corresponding author by request.

\section{Conflicts of Interest}

The authors declare that there are no conflicts of interest regarding the publication of this paper.

\section{Acknowledgments}

This work was supported by the Hubei Key Laboratory of Disaster Prevention and Mitigation (China Three Gorges University) Open Research Program (no. 2016KJZ07), the CRSRI Open Research Program (no. CKWV2019755/KY) and the Chinese National Natural Science Foundation (nos. 51909137 and 51809212).

\section{References}

[1] S. A. Shivvakumar, Shivamanth, C. H. Solanki, and G. R. Dodagoudar, "Seepage and Stability analyses of earth dam using finite element method," Aquatic Procedia, vol. 4, pp. 876-883, 2015.

[2] T. A. Middlebrooks, "Earth-dam practice in United States," Transactions of American Society Civil Engineering, vol. 118, pp. 697-722, 1953.

[3] E. Loukola, P. Reiter, C. Shen et al., "Embankment dams and their foundation: evaluation of erosion," in Proceedings of the International Workshop on Dam Safety Evaluation, pp. 171-188, Grindewald, Switzerland, 1993.

[4] X. Q. Niu, "Characteristics of reservoir defects and rehabilitation technology in China," Chinese Journal of Geotechnical Engineering, vol. 32, no. 1, pp. 153-157, 2010.

[5] Y. L. Li, S. Y. Li, X. F. Zhang, and R. Fan, "Research on bypass seepage of dam abutment deep-thickness sand layer and anti-seepage scheme," in Proceedings of the IEEE 2010 AsiaPacific Power and Energy Engineering Conference, pp. 1-4, Chengdu, China, March 2010.

[6] T. Jiang, J. Zhang, W. Wan, S. Cui, and D. Deng, "3D transient numerical flow simulation of groundwater bypass seepage at the dam site of Dongzhuang hydro-junction," Engineering Geology, vol. 231, pp. 176-189, 2017.

[7] H. Wei and Z. Z. Shen, "Reliability analysis on seepage stability of earth dams and its application," Chinese Journal of Geotechnical Engineering, vol. 30, pp. 1404-1409, 2008.

[8] J. H. Li, Y. Wang, and Q. Hu, "Probability analysis of seepage failure of embankments based on stochastic finite element method," Rock and Soil Mechanics, vol. 27, no. 10, pp. 1847-1850, 2006.

[9] R. Hu, Y. F. Chen, D. Q. Li et al., "Reliability analysis of seepage stability of core-wall rockfill dam based on stochastic response surface method," Rock and Soil Mechanics, vol. 33, pp. 1051-1060, 2012.

[10] S. H. Jiang, D. Q. Li, C. B. Zhou, and L. M. Zhang, "Capabilities of stochastic response surface method and response surface method in reliability analysis," Structural Engineering and Mechanics, vol. 49, no. 1, pp. 111-128, 2014.

[11] S. H. Jiang, D. Q. Li, L. M. Zhang, and C. B. Zhou, "Slope reliability analysis considering spatially variable shear strength parameters using a non-intrusive stochastic finite element method," Engineering Geology, vol. 168, pp. 120-128, 2014.

[12] S. L. Pan, Q. F. Wang, and J. Yu, "Improvement of analysis of free surface seepage problem by using initial flow method," Chinese Journal of Geotechnical Engineering, vol. 34, no. 2, pp. 202-209, 2012.

[13] H. D. Cui and Y. M. Zhu, "Improved procedure of nodal virtual flux of global iteration to solve seepage free surface," Journal of Wuhan University of Technology (Transportation Science \& Engineering), vol. 33, no. 2, pp. 238-241, 2009.

[14] Y. Chen, R. Hu, C. Zhou, D. Li, and G. Rong, "A new parabolic variational inequality formulation of Signorini's condition for non-steady seepage problems with complex seepage control systems," International Journal for Numerical and Analytical Methods in Geomechanics, vol. 35, no. 9, pp. 1034-1058, 2011.

[15] J. W. He, G. M. Liu, and W. Qiao, "Equivalent nodal flow method used to determine overflow surface in seepage with free surface," Journal of China Three Gorges University (Natural Sciences), vol. 35, pp. 34-37, 2013.

[16] S. S. Isulapalli, A. Roy, and P. G. Georgopoulos, "Stochastic response surface methods for uncertainty propagation: application to environmental and biological systems," Risk Analysis, vol. 18, no. 3, pp. 351-363, 1998.

[17] H. P. Gavin and S. C. Yau, "High-order limit state functions in the response surface method for structural reliability analysis," Structural Safety, vol. 30, no. 2, pp. 162-179, 2008.

[18] M. R. Rajashekhar and B. R. Ellingwood, "A new look at the response surface approach for reliability analysis," Structural Safety, vol. 12, no. 3, pp. 205-220, 1993. 
[19] H. S. Li, Research on Probability Uncertainty Analysis and Design Optimization Method, Northwestern Polytechnical University, Xi'an, China, 2008.

[20] Y. Noh, K. K. Choi, and L. Du, "Reliability-based design optimization of problems with correlated input variables using a Gaussian Copula," Structural and Multidisciplinary Optimization, vol. 38, no. 1, pp. 1-16, 2009.

[21] P.-L. Liu and A. Der Kiureghian, "Multivariate distribution models with prescribed marginals and covariances," Probabilistic Engineering Mechanics, vol. 1, no. 2, pp. 105-112, 1986.

[22] D. Z. Wen, R. H. Zhuo, D. J. Ding, H. Zheng, J. Cheng, and Z.-H. Li, "Generation of correlated pseudo random variables in Monte Carlo simulation," Acta Physica Sinica, vol. 61, no. 22, pp. 1-8, 2012.

[23] I. Kaymaz and C. A. McMahon, "A response surface method based on weighted regression for structural reliability analysis," Probabilistic Engineering Mechanics, vol. 20, no. 1, pp. 11-17, 2004.

[24] Z. Z. Lv, S. F. Song, H. S. Li et al., Structural Mechanism Reliability and Reliability Sensitivity Analysis, Science Press, Beijing, China, 2009. 


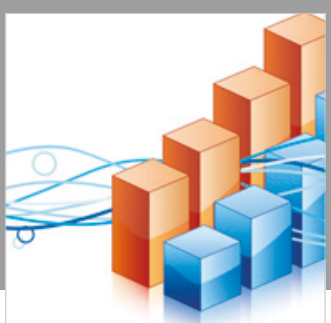

Advances in

Operations Research

\section{-n-m}
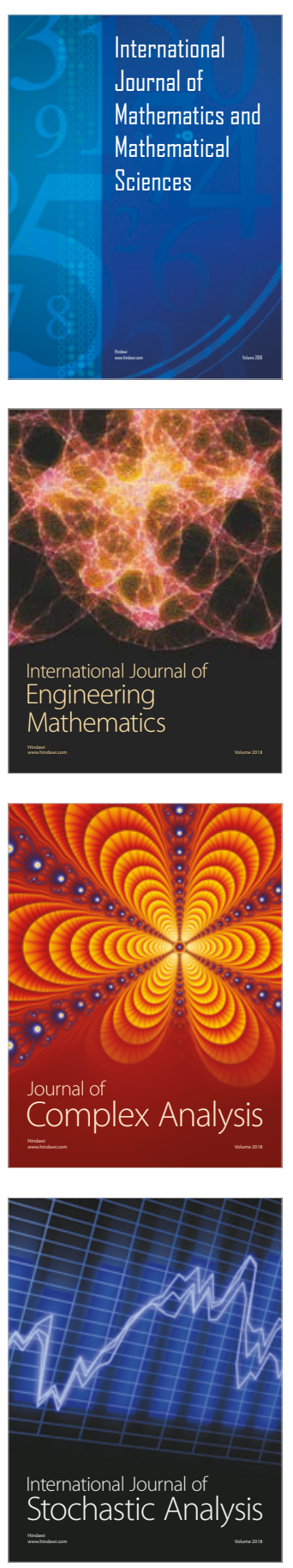
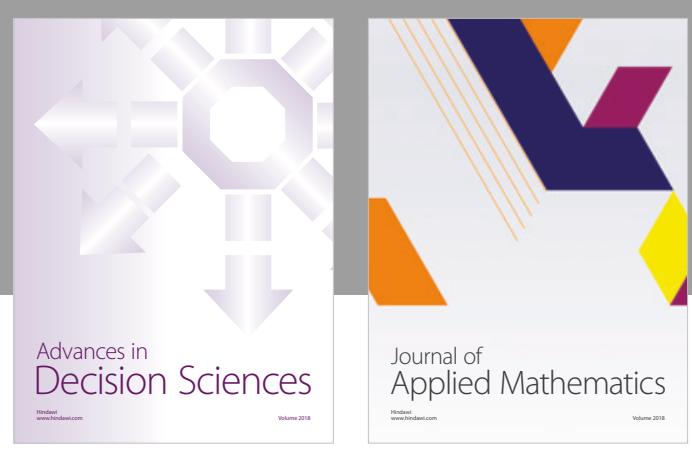

Journal of

Applied Mathematics


Submit your manuscripts at

www.hindawi.com

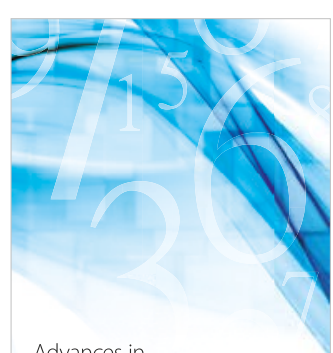

Advances in
Numerical Analysis
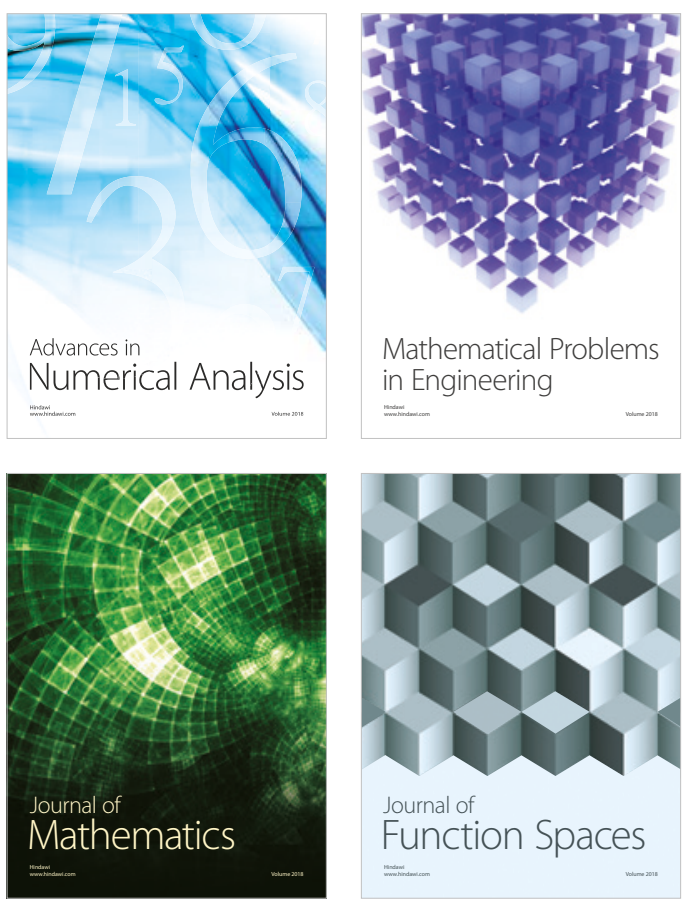

Mathematical Problems in Engineering

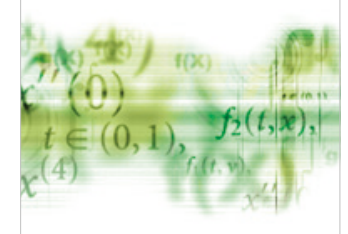

International Journal of

Differential Equations

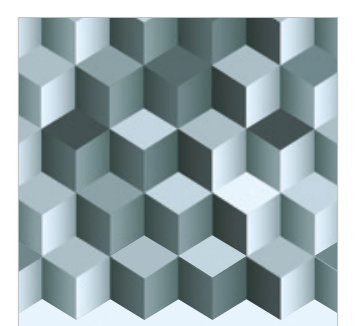

Journal of

Function Spaces

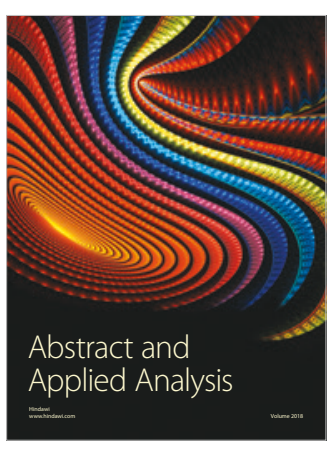

The Scientific

World Journal

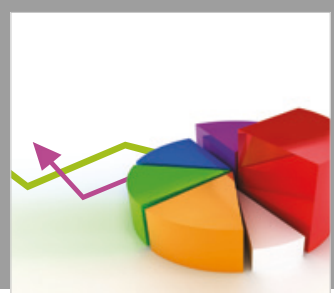

Journal of

Probability and Statistics
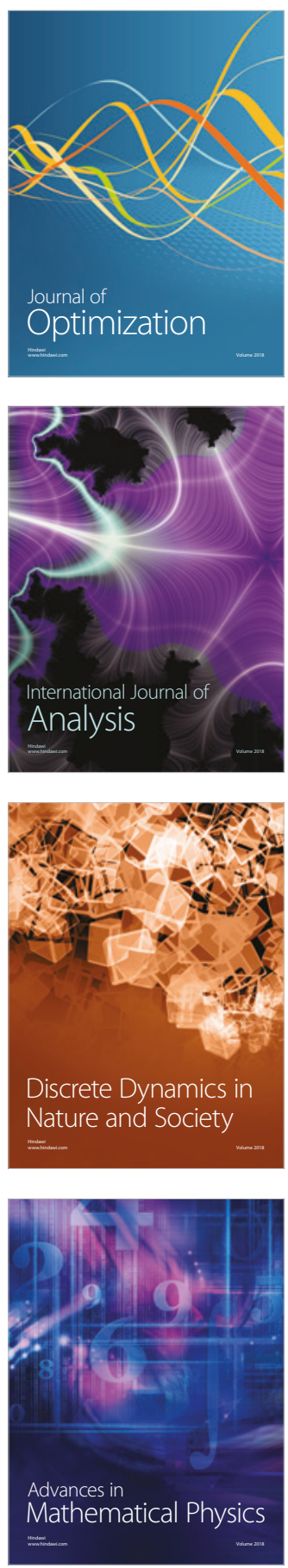